\title{
Stellar activity, differential rotation, and exoplanets
}

\author{
A. F. Lanza \\ INAF-Osservatorio Astrofisico di Catania, Via S. Sofia, 78, 95123 Catania, Italy \\ email: nuccio.lanza@oact.inaf.it
}

\begin{abstract}
The photospheric spot activity of some of the stars with transiting planets discovered by the CoRoT space experiment is reviewed. Their out-of-transit light modulations are fitted by a spot model previously tested with the total solar irradiance variations. This approach allows us to study the longitude distribution of the spotted area and its variations versus time during the five months of a typical CoRoT time series. The migration of the spots in longitude provides a lower limit for the surface differential rotation, while the variation of the total spotted area can be used to search for short-term cycles akin the solar Rieger cycles. The possible impact of a close-in giant planet on stellar activity is also discussed.
\end{abstract}

Keywords. Stars: activity; stars: magnetic fields; stars: spots; stars: rotation; planetary systems

\section{Introduction}

Magnetic fields in the Sun and late-type stars produce active regions in the photosphere by affecting the transfer of energy and momentum in the outermost convective layers. These features consist of dark spots, bright faculae, and an enhanced network of magnetic flux tubes with radii down to about $100 \mathrm{~km}$ and $\mathrm{kG}$ field strength. Solar active regions can be studied in details thanks to a spatial resolution down to $\sim 100 \mathrm{~km}$ and a time resolution better than $\sim 1 \mathrm{~s}$.

In distant stars, we lack spatial resolution (with the exception of the supergiant $\alpha$ Orionis, see Dupree (2011)), so we must apply indirect techniques to map their photospheres. The most successful is Doppler Imaging that produces a two-dimensional map of the surface from a sequence of high-resolution line profiles, sampling the different rotation phases of the star (see, e.g., Strassmeier $(2009,2011)$ ). Its application requires that the rotational broadening of the spectral lines exceeds the macroturbulence by at least a factor of $4-5$, implying a minimum $v \sin i \sim 10-15 \mathrm{~km} \mathrm{~s}^{-1}$ in dwarf stars. Therefore, late-type stars that rotate as slowly as the Sun cannot be imaged by this technique. For those stars, we derive the distribution of brightness inhomogeneities from the rotational modulation of the flux produced by cool spots and bright faculae that come into and out of view as the star rotates. By comparing successive rotations, it is possible to study the time evolution of the active regions.

Another method exploits the tiny light modulations produced by the occultation of starspots by a transiting giant planet moving in front of the disc of its star (e.g. SilvaValio et al. (2010); Wolter et al. (2009)). It is a specialized version of the eclipse mapping technique, developed for the active components of close binary stars, e.g., Collier Cameron (1997); Lanza et al. (1998). Its application will be reviewed by Silva-Valio (2011). Stellar differential rotation and activity cycles can be studied also through techniques of time series analysis applied to sequences of seasonal optical photometry; see, e.g., Messina \& Guinan (2003); Kolláth \& Oláh (2009); Oláh (2011). 
In this review, I shall briefly report on the modelling of the light curves of some planethosting stars as observed by the space experiment CoRoT.

\section{Space-borne optical photometry}

Space-borne optical photometry has provided time series for several late-type stars spanning from several days up to several months thanks to the space missions MOST (Walker et al. 2003), CoRoT (Auvergne et al. 2009), and Kepler (see, e.g., Ciardi et al. 2011).

MOST (the Microvariability and Oscillation of Stars satellite) has a telescope of $15 \mathrm{~cm}$ aperture that can observe a given target for up to $40-60$ days reaching a photometric precision of $50-100 \mathrm{ppm}$ (parts per million) on the brightest stars $(V \leqslant 4)$. It has observed $\epsilon$ Eridani (Croll et al. 2006) and $\mathrm{k}^{1}$ Ceti (Walker et al. 2007) whose light curves have been modelled to extract information on stellar differential rotation.

CoRoT (Convection, Rotation and Transits) is a space experiment devoted to asteroseismology and the search for extrasolar planets through the method of transits. With its 27-cm aperture telescope, it can observe up to 12,000 targets per field for intervals of 150 days searching for transit signatures in the light curves. Its white-band light curves have a sampling of 32 or $512 \mathrm{~s}$ and a bandpass ranging from 300 to $1100 \mathrm{~nm}$. A photometric accuracy of $\sim 100 \mathrm{ppm}$ is achieved for a $\mathrm{G}$ or K-type star of $V \sim 12$ by integrating the flux over individual orbits of the satellite. CoRoT provides some chromatic information on the light variability of its brightest targets $(V<14.5)$.

Kepler, launched in March 2009, has a telescope of $95 \mathrm{~cm}$ aperture and is continuously monitoring $\sim 100,000$ dwarfs in a fixed field of view for a time interval of at least 3.5 years, searching for planetary transits. Its accuracy reaches $\sim 30 \mathrm{ppm}$ in 1 hour integration on a G2V target of $V \sim 12$.

Stars with transiting giant planets allow us to derive the inclination of the stellar rotation axis, which is important to modelling their light curves for stellar activity studies (see, e.g., Mosser et al. (2009)). Specifically, by fitting the transit light curve, we can measure the inclination of the planetary orbit along the line of sight, which is equal to the inclination of the stellar rotation axis if the spin and the orbital angular momentum are aligned. This hypothesis can be tested with the so-called Rossiter-McLaughlin effect, i.e., the apparent anomaly in the radial velocity of the star observed during the planetary transit that allows us to measure the angle between the projections of the stellar spin and the orbital angular momentum on the plane of the sky (Winn et al. 2005). Another advantage of stars with transiting planets is that their fundamental parameters have been well determined because accurate stellar masses and radii are needed to derive accurate planetary parameters from the transit modelling.

\section{Light curve modelling}

Spot models of the light curves of late-type stars observed by MOST and CoRoT have already been published, while the modelling of Kepler light curves has just started (Brown et al. 2011). MOST time series were exploited to measure stellar differential rotation by fitting the wide-band light modulation with a few circular spots with fixed contrast. Thanks to such assumptions, it was possible to derive the latitudes of the spots and measure the variation of the angular velocity vs. latitude (Croll et al. 2006; Walker et al. 2007). For a generalization of that approach with the CoRoT light curves, see Mosser et al. (2009). 
In the Sun, active regions consist not only of cool spots but also of bright faculae whose contrast is maximum close to the limb and minimum at the disc centre. Moreover, the optical variability of the Sun is dominated by several active regions at the same time making a model based on a few spots poorly suitable to reproduce its active region pattern.

Lanza et al. (2007) used the time series of the Total Solar Irradiance (TSI, e.g., Fröhlich \& Lean (2004)) as a proxy for the solar optical light curve to test different modelling approaches assuming that the active regions consist of dark spots and bright faculae with fixed contrasts and in a fixed area proportion. A model with a continuous distribution of active regions and the maximum entropy regularization, to warrant the uniqueness and stability of the solution, is the most suitable and reproduces the distribution of the area of the sunspot groups vs. longitude with a resolution better that $\sim 50^{\circ}$, as well as the variation of the total spotted area vs. time. It allows a highly accurate reproduction of the TSI variations with a typical standard deviation of the residuals of $\sim 30-35 \mathrm{ppm}$ for time intervals of 14 days. However, the value of the faculae-to-spotted area ratio is a critical parameter because it affects the derived distribution of the active regions vs. longitude (see, Lanza et al. (2007), for a detailed discussion).

The maximum entropy spot model tested in the case of the Sun has been applied to CoRoT light curves to derive the distribution of the stellar active regions vs. longitude and the variation of their total area vs. time. In general, information on the latitudes of stellar active regions cannot be extracted from a one-dimensional data set such as an optical light curve. Moreover, since the inclination of the rotation axis of the stars with transiting planets is generally close to $90^{\circ}$, it is impossible to constrain the spot latitudes because the duration of the transit of a spot across the stellar disc is independent of its latitude.

\section{Results from CoRoT light curves}

Lanza et al. (2009a) model the out-of-transit light curve of CoRoT-2, a G7V star with a giant planet with a mass of 3.3 Jupiter masses and an orbital period of 1.743 days (Alonso et al. 2008; Bouchy et al. 2008). Since the spot pattern is evolving rapidly, they model individual intervals of 3.15 days along a sequence of 142 days. The star has a light curve amplitude of $0.06 \mathrm{mag}$, i.e., about 20 times that of the Sun at the maximum of the 11-yr cycle. Solar-like contrasts for the spots and the faculae are adopted.

The distribution of the spotted area vs. longitude and time is plotted in Fig. 4 of Lanza et al. (2009a), here reproduced in Fig. 1. The active regions are mainly found within two active longitudes, initially separated by $\sim 180^{\circ}$. The longitude initially around $0^{\circ}$ does not migrate, i.e., it rotates with the same period as the adopted reference frame, while the other longitude, initially around $180^{\circ}$, migrates backward, i.e., it rotates slower than the reference frame by $\sim 0.9$ percent. Individual active regions also migrate backward as they evolve, i.e., they rotate slower than the active longitude to which they belong, with a maximum difference of $\approx 2$ percent. The relative migration of the active longitudes can be interpreted as a consequence of their different latitudes on a differentially rotating star, yielding a lower limit of 0.9 percent for the amplitude of the differential rotation (Lanza et al. 2009a). On the other hand, the backward migration of the individual active regions can be regarded as analogous to the braking of the rotation of solar active regions as they evolve because the relative amplitude of the angular velocity variation is remarkably similar e.g., (Zappalà \& Zuccarello 1991; Schüssler \& Rempel 2005).

Other authors have suggested a greater amplitude for the differential rotation of CoRoT-2, up to $\sim 8$ percent, from the migration of individual active regions (Fröhlich 


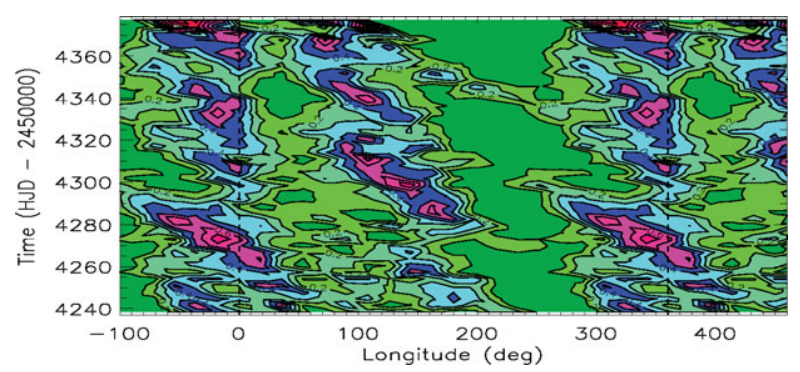

Figure 1. The isocontours of the spot filling factor vs. longitude and time for CoRoT-2 after Lanza et al. (2009a). The longitude reference frame rotates with the star with a period of 4.5221 days and the longitude increases in the same direction of the stellar rotation. The longitude scale has been extended beyond $0^{\circ}$ and $360^{\circ}$ (marked by the vertical dashed lines) to help follow the migration of the spots. In the electronic version, different colours indicate different relative filling factors of the starspots; from the minimum to the maximum: green, light green, light blue, blue, light pink, pink, and red.

et al. 2009; Huber et al. 2010). Savanov (2010) notices that the active regions appear in an alternate way in the two active longitudes, suggesting a short-term flip-flop phenomenon, reminiscent of the flip-flop cycles in some active stars that, however, have timescales of several years (Berdyugina \& Tuominen 1998; Berdyugina 2005).

The variation of the total spotted area shows remarkable oscillations with a cycle of $\sim 29$ days (see Fig. 6 of Lanza et al. (2009a)). In the Sun, short-term oscillations of the total spotted area have been observed close to the maximum of some of the 11-yr cycles and are called Rieger cycles. They have periods around 160 days, i.e., about five times longer than the cycles observed in CoRoT-2 (Oliver et al. 1998; Krivova \& Solanki 2002; Zaqarashvili et al. 2010). Lou (2000) suggests that they may be due to hydromagnetic Rossby-type waves trapped in the solar convection zone. Since the wave frequency is proportional to the rotation frequency of the star, the expected period is close to that observed in CoRoT-2 because this star rotates five times faster than the Sun.

The approach introduced for CoRoT-2 has been applied to other late-type planethosting stars, viz. CoRoT-4 (Lanza et al. 2011), CoRoT-6 (Lanza et al. 2010), and CoRoT-7 (Lanza et al. 2011). From the migration of their active longitudes, a lower limit for the amplitude of their latitudinal differential rotation has been obtained. The results are listed in Table 1, together with those derived from MOST photometry and those by Mosser et al. (2009) for two of the CoRoT asteroseismic targets. In Table 1, the columns from left to right list the name of the star, its effective temperature $T_{\text {eff }}$, its mean rotation period $P_{\text {rot }}$, the relative amplitude of the differential rotation $\Delta \Omega / \Omega$, and the references.

A comparison with the differential rotation amplitudes as derived from Doppler Imaging or the Fourier transform of the spectral line profiles (Reiners (2006)) shows that the values derived from the migration of the active longitudes are generally smaller than those expected in stars with the same effective temperature and rotation rates, by a typical factor of $2-3$. This suggests that their active regions are mostly localized at low latitudes, as in the case of the Sun.

\section{The possible case for a magnetic star-planet interaction}

The planets of CoRoT-2, CoRoT-4, and CoRoT-6 are hot Jupiters, i.e., giant planets orbiting within 0.15 AU from their host stars. They interact tidally and possibly magnetically with their stars, which may lead to observable effects on stellar activity (see, 
Table 1. Stellar differential rotation from spot modelling of space-borne photometry.

\begin{tabular}{|c|c|c|c|c|}
\hline Star & $\begin{array}{l}T_{\text {eff }} \\
(\mathrm{K})\end{array}$ & $\begin{array}{c}P_{\text {rot }} \\
\text { (days) }\end{array}$ & $\Delta \Omega / \Omega$ & References \\
\hline$\epsilon$ Eridani & 4830 & 11.45 & $0.11 \pm 0.03^{*}$ & (Croll et al. 2006, Croll et al. (2006)) \\
\hline CoRoT-7 & 5275 & 23.64 & $0.058 \pm 0.017$ & (Lanza et al. $2010 \mathrm{~b}$, Lanza et al. $(2010 \mathrm{~b})$ ) \\
\hline $\mathrm{k}^{1}$ Ceti & 5560 & 8.77 & $0.09 \pm 0.006^{*}$ & (Walker et al. 2007, Walker et al. (2007)) \\
\hline CoRoT-2 & 5625 & 4.52 & $\sim 0.009-0.08$ & $\begin{array}{l}\text { (Lanza et al. } 2009 \text { a, Lanza et al. }(2009 a) \text { ) } \\
\text { (Fröhlich et al. } 2009 \text {. Fröhlich et al. } 2009) \text { ) }\end{array}$ \\
\hline HD 175726 & 6030 & 3.95 & $\approx 0.40^{*}$ & (Mosser et al. 2009, Mosser et al. (2009)) \\
\hline CoRoT-6 & 6090 & 6.35 & $0.12 \pm 0.02$ & (Lanza et al. 2010a, Lanza et al. (2010a)) \\
\hline CoRoT-4 & 6190 & 9.20 & $0.057 \pm 0.015$ & (Lanza et al. 2009b, Lanza et al. (2009b)) \\
\hline HD 181906 & 6360 & 2.71 & $\approx 0.25^{*}$ & (Mosser et al. 2009, Mosser et al. (2009)) \\
\hline
\end{tabular}

Note:

${ }^{*}$ Value of the $K$ coefficient estimated for a solar-like differential rotation law $P(\phi)=P_{\text {eq }} /\left(1-K \sin ^{2} \phi\right)$, where $\phi$ is the latitude, $P(\phi)$ the rotation period at latitude $\phi$, and $P_{\mathrm{eq}}$ the rotation period at the equator.

e.g., Cuntz et al. 2000; Lanza 2008, 2009). Current evidence of star-planet magnetic interaction (hereafter SPMI) is limited to the modulation of the chromospheric flux with the orbital phase of the planet in a few stars and in some seasons (Shkolnik et al. (2005, 2008)). Evidence of a coronal flux enhacement is much more controversial (cf. Kashyap et al. 2008 and Poppenhaeger et al. 2010), although some possible cases have been presented (Saar et al. (2008); Pillitteri et al. (2010)). SPMI features in the photosphere have been proposed for $\tau$ Bootis (Walker et al. 2008), CoRoT-2 (Pagano et al. 2009), and, possibly, HD 192263 (Santos et al. 2003). The mean rotation of $\tau$ Boo is synchronized with the orbital motion of its giant planet, so a modulation of its optical flux with the orbital period of the planet cannot be unambiguously attributed to SPMI. Nevertheless, Walker et al. (2008) found an active region on the star which lasted for at least $\sim 500$ stellar rotations, i.e., 5 years, always leading the subplanetary meridian by $\sim 70^{\circ}$. The persistence of such a feature strongly suggests a connection with the planet.

Lanza et al. (2009b) suggest a similar phenomenon in the other synchronous system CoRoT-4, finding an active region located at the subplanetary longitude that has persisted for $\sim 70$ days. Even more intriguing is the case of CoRoT-6, a non-synchronous system with a planetary orbital period of 8.886 days and a mean stellar rotation period of 6.35 days. Assuming a longitude reference frame rotating with the mean stellar rotation period, the maximum of the spot filling factor in several active regions occurs when they cross a meridian at $-200^{\circ}$ from the subplanetary meridian. The probability of a chance occurrence is only $\sim 0.8$ percent (Lanza et al. (2010a)).

It is difficult to find a mechanism for the allegedly supposed influence of the planet on the formation of stellar active regions. Lanza (2008) conjectured that the reconnection of the stellar coronal field with the magnetic field of the planet may induce a longitudal dependence of the hydromagnetic dynamo action in the star, provided that some of the spot magnetic flux tubes come from the subphotospheric layers, as suggested by Brandenburg (2005). Nevertheless, further observations of the photospheric SPMI are needed firstly to confirm the reality of the phenomenon and secondly to derive its dependence on stellar and planetary parameters.

\section{References}

Aigrain, S., et al. 2008, Astron. Astrophys, 488, L43

Alonso, R., et al. 2008, Astron. Astrophys, 482, L21

Auvergne, M., et al. 2009, Astron. Astrophys, 506, 411 
Berdyugina, S. V. 2005, Living Reviews in Solar Physics, 2, 8

Berdyugina, S. V. \& Tuominen, I. 1998, Astron. Astrophys, 336, L25

Bouchy, F., et al. 2008, Astron. Astrophys, 482, L25

Brown, A., Korhonen, H., Berdyugina, S, et al., 2011, these proceedings

Brandenburg, A. 2005, Astrophys. J., 625, 539

Ciardi, D. R., van Braun, K., Bryden, G., et al. 2011, Astronomical J., 141, 108

Collier Cameron, A. 1997, MNRAS, 287, 556

Croll, B., et al. 2006, Astron. Astrophys, 648, 607

Cuntz, M., Saar, S. H., \& Musielak, Z. E. 2000, Astrophys. J., 533, L151

Dupree, A. 2011, these proceedings

Fröhlich, H.-E., Küker, M., Hatzes, A. P., \& Strassmeier, K. G. 2009, Astron. Astrophys, 506, 263

Fröhlich, C. \& Lean, J. 2004, A\& AR, 12, 273

Haas, M. R., et al. 2010, Astrophys. J., 713, L115

Huber, K. F., Czesla, S., Wolter, U., \& Schmitt, J. H. M. M. 2010, Astron. Astrophys, 514, A39

Kashyap, V. L., Drake, J. J., \& Saar, S. H. 2008, Astrophys. J., 687, 1339

Kolláth, Z. \& Oláh, K. 2009, Astron. Astrophys, 501, 695

Krivova, N. A. \& Solanki, S. K. 2002, Astron. Astrophys, 394, 701

Lanza, A. F. 2008, Astron. Astrophys, 487, 1163

Lanza, A. F. 2009, Astron. Astrophys, 505, 339

Lanza, A. F., Catalano, S., Cutispoto, G., Pagano, I., \& Rodono, M. 1998, Astron. Astrophys, 332,541

Lanza, A. F., Bonomo, A. S., \& Rodonò, M. 2007, Astron. Astrophys, 464, 741

Lanza, A. F., et al. 2009a, Astron. Astrophys, 493, 193

Lanza, A. F., et al. 2009b, Astron. Astrophys, 506, 255

Lanza, A. F., et al. 2010, Astron. Astrophys, 520, A53

Lanza, A. F., et al. 2011, Astron. Astrophys, 525, A14

Lou, Y.-Q. 2000, Astrophys. J., 540, 1102

Messina, S. \& Guinan, E. F. 2003, Astron. Astrophys, 409, 1017

Mosser, B., Baudin, F., Lanza, A. F., et al. 2009, Astron. Astrophys, 506, 245

Oláh, K. 2011, these proceedings

Oliver, R., Ballester, J. L., \& Baudin, F. 1998, Nature, 394, 552

Pagano, I., Lanza, A. F., Leto, G., et al. 2009, Earth, Moon, and Planets, 105, 373

Pillitteri, I., Wolk, S. J., Cohen, O. et al. 2010, Astrophys. J., 722, 1216

Poppenhaeger, K., Robrade, J., \& Schmitt, J. H. M. M. 2010, Astron. Astrophys, 515, A98

Reiners, A. 2006, Astron. Astrophys, 446, 267

Saar, S. H., Cuntz, M., Kashyap, V. L., \& Hall, J. C. 2008, IAU Symposium, 249, 79

Santos, N. C., et al. 2003, Astron. Astrophys, 406, 373

Savanov, I. S. 2010, Astron. Rep., 54, 437

Schüssler, M. \& Rempel, M. 2005, Astron. Astrophys, 441, 337

Shkolnik, E., et al. 2005, Astrophys. J., 622, 1075

Shkolnik, E., Bohlender, D. A., Walker, G. A. H., \& Collier Cameron, A. 2008, Astrophys. J., 676,628

Silva-Valio, A. 2011, these proceedings

Silva-Valio, A., Lanza, A. F., Alonso, R., \& Barge, P. 2010, Astron. Astrophys, 510, A25

Strassmeier, K. G., 2009, A\&AR, 17, 251

Strassmeier, K. G., 2011, these proceedings

Walker, G., et al. 2003, PASP, 115, 1023

Walker, G. A. H., et al. 2007, Astrophys. J., 659, 1611

Walker, G. A. H., et al. 2008, Astron. Astrophys, 482, 691

Winn, J. N., et al. 2005, Astrophys. J., 631, 1215

Wolter, U., Schmitt, J. H. M. M., Huber, K. F., et al. 2009, Astron. Astrophys, 504, 561

Zappalà, R. A. \& Zuccarello, F. 1991, Astron. Astrophys, 242, 480

Zaqarashvili, T. V., Carbonell, M., Oliver, R., \& Ballester, J. L. 2010, Astrophys. J., 709, 749 


\section{Discussion}

K. Strassmeier: First of all, this is a very excellent idea to verify this with whitelight photometry of the irradiance, but it does also ring a little bit of a warning bell because, for example, I presume - and please do correct me - that you assume a faculae component and a spot component at the same time. The time scales, the lifetime of these two features are markedly different; but you implicitly assume they are living the same time and actually give the same constant contribution. But if you have a different lifetimes, they would actually give a variable contribution.

LANZA: Yes, you are right. Faculae are a possible source of problems in this kind of modeling. If the star is very active, probably the cool spots dominate the variation. When we consider the stars that have the same level of activity as the sun, faculae may be a problem; and also the longitudinal distribution of the spots may be affected. It is difficult for the Sun to have a good treatment of the faculae because, as you mentioned, they evolve on different timescales. The ratio between the faculae and the spotted area may change during the evolution of an active region. So this model is very simplified. It assumes a constant ratio, and that may affect both the evolution of the area and also that of the longitude positions.

A. Kosovichev: Antonino, interesting study. I'm wondering if your technique estimates the differential rotation for slow-rotating stars like the sun?

LANZA: We focus on stars with exoplanets because those are the targets with the better characterization, and we also have sometimes information on the inclination of the rotation axis. Certainly in the CoRoT-4 data bases, there are a lot of star that's rotate as slowly as the sun or even slower. But as far as I know, no planets have been found for those stars. And also another problem is that the time series is quite limited. So in 150 days, you have only a few rotations; and that may be difficult to trace the migration accurately. So we focused on stars that rotate faster than the sun because of these two limitations, but certainly it is a very interesting question, and it deserves more attention for the future. 